\title{
Las tierras y territorios rurales como escenarios funcionales para la práctica de la Economía Popular y Solidaria en Ecuador: algunos elementos sobre su uso y aprovechamiento
}

\section{Land and rural territories as functional sceneries for the practi- ce of popular solidarity economy in Ecuador: some elements on their use}

\author{
Carlos Nieto C. ${ }^{1}$, Azucena Vicuña ${ }^{1}$

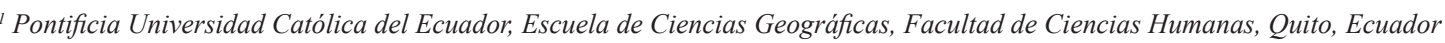

\begin{abstract}
Resumen
El artículo presenta un estudio analítico sobre la caracterización del uso y del potencial productivo de los territorios y tierras rurales en Ecuador, como escenario para la aplicación del modelo de Economía Popular y Solidaria, EPS. Para el desarrollo de la investigación, se planteó la siguiente hipótesis: "La posesión y usufructo de una parcela agrícola, individual o colectiva en las áreas rurales donde prevalece la presencia de los actores de la Economía Popular y Solidaria, apenas facilita su sobrevivencia, pero no es garantía para una reproducción familiar apropiada o para una vida familiar digna". Con base en los resultados de la investigación, la hipótesis fue aceptada como verdadera. Uno de los principales resultados fue la relación directa entre la pobreza de las poblaciones y su actividad económica principal, que es agricultura, aplicada en tierras con aptitud natural para forestación o conservación. Además, se encontró que las actividades productivas de las poblaciones rurales actoras de la EPS, están sujetas a riesgos constantes, en función de las amenazas de eventos climáticos adversos y de la vulnerabilidad de las áreas en producción, por estar ubicadas en ecosistemas frágiles. Por otro lado, se encontró que el tamaño promedio de Unidades Productivas, Agropecuarias, UPA, está muy por debajo del tamaño mínimo requerido para hacer agricultura redituable y la tendencia a continuar subdividiendo las UPA, es creciente. Sin embargo, aun con estos tamaños micro de UPA, las familias dejan de cultivar parte de sus fundos o cambian de uso productivo desde cultivos a pastos para cría de ganado, esto lo hacen para evitar el uso de la mano de obra en actividades agrícolas no rentables en la UPA y ofertar la mano de obra en actividades remuneradas extra finca. Las conclusiones del estudio fueron: i) Tanto por la calidad de la tierra o territorio (aptitud natural de uso), como por la cantidad disponible (tamaño de UPA insuficiente), la posesión y usufructo de una UPA, no es una garantía para la reproducción y aseguramiento de la calidad de vida familiar, en la mayoría de áreas rurales de la Sierra ecuatoriana, pero especialmente para las comunidades y grupos indígenas, que se supone conforman la EPS; ii) En muchos casos, la tierra como pertenencia individual o colectiva, más bien se ha convertido en el principal y acaso único activo del patrimonio familiar o comunal, antes de ser el medio de producción, y iii) Se ha evidenciado que el tipo de agricultura denominada "agricultura familiar", "agricultura de subsistencia", o "agricultura de sobrevivencia", todavía reinvindicada por varios investigadores y promotores rurales, parecería no tener un futuro prometedor.
\end{abstract}

Palabras clave: economía popular y solidaria; tierras y territorios rurales; aptitud natural de uso del suelo; aprovechamiento del suelo; tamaño de UPA. 


\begin{abstract}
This paper is an analytical revision and characterization of the usefulness and productive potential of rural lands and territories in Ecuador, as a scenery to apply the Popular and Solidary Economy model, (PSE, in Spanish). The study was developed under the following research hypothesis: "The ownership and use of agricultural land, in rural areas, where there is prevalence of the Popular and Solidary Economy actors, could perhaps facilitate their daily surviving, but it is not a guarantee for a suitable family reproduction or a worthy living style". Based on the research results, the hypothesis was accepted as being true. One of the primary findings was the direct relationship between poverty of the populations and its main income activity, which is farming done in lands apt for forestry and conservation, but not for agriculture. Additionally, it was found that the agricultural productive systems of PSE groups are constantly at risk, in reference to adverse climate threats combined with the vulnerability of their geographic location in fragile ecosystems. The study also showed that the average size of an Agricultural Land Productive Unit, (UPA, in Spanish), is considerably below the minimum required to obtain profitable family incomes and there is an increasing tendency to split up it, even more. However the micro size of UPA, the families do not cultivate the whole available land, or they change the land use, from crops to grasses; this is mainly to liberate labor from a not profitable labor demanding crops and to devote this labor to remunerated extra farm activities. The main conclusions were: i) Because of both, the quality of agricultural land (natural aptitude of use) and for the availability of land (insufficient UPA size), the ownership and usufruct of an UPA means no guarantee for the reproduction and to assurance quality of life in the majority of the rural families in Ecuador. This is especially true for the communities and indigenous groups who supposedly are PSE groups; ii) In many cases, the land, either as a private or collective ownership, has become the main and even the only family or community asset instead of being the mean of production; and iii) It is evident that the type of agriculture known as "family agriculture", "self-sustaining agriculture" or "Self-surviving agriculture", still claimed by several researchers and rural develop promoters, does not have a promising future anymore.
\end{abstract}

Keywords: popular and solidary economy; land and territories; land natural aptitude of use; land usefulness; size of UPA.

\section{Introducción}

Para entender el papel de las tierras y territorios rurales como escenarios del modelo de Economía Popular y Solidaria, EPS, es necesario rescatar su definición oficial: "La Economía Popular y Solidaria, es la forma de organización económica, donde sus integrantes, individual o colectivamente, organizan y desarrollan procesos de producción, intercambio, comercialización, financiamiento y consumo de bienes y servicios, para satisfacer necesidades $y$ generar ingresos, basadas en relaciones de solidaridad, cooperación y reciprocidad, privilegiando al trabajo y al ser humano como sujeto y fin de su actividad, orientada al Buen Vivir, en armonía con la naturaleza, por sobre la apropiación, el lucro y la acumulación de capital"; artículo 1, Ley Orgánica de Economía Popular y Solidaria (Asamblea Nacional del Ecuador, 2011). Al margen de cualquier inter- pretación del concepto, es obvio que esta forma de organización económica, por lo menos en lo que se refiere al ámbito rural y basada en la producción de bienes primarios, si ha de lograrse, necesariamente debe ser en un espacio territorial con aptitudes para la producción y con sentido de dominio o pertenencia, si bien esta pertenencia no fuera con sentido de apropiación, para ser consecuentes con la definición de EPS; aunque para otros autores, la EPS en realidad no tiene modelos, o no hay un sendero cierto y lineal que pueda mapearse y proveer una ruta programada de acción, lo cual eventualmente puede justificar la pertenencia o incluso la apropiación de los territorios como escenarios de la EPS (Coraggio, sf).

Un componente importante del Objeto de la Ley de EPS es: Potenciar las prácticas que se desarrollan en las comunas, comunidades, pueblos y nacionalidades, y en sus unidades económicas pro- 
ductivas para alcanzar el Sumak Kawsay; literal b, artículo 3, de la Ley Orgánica de Economía Popular y Solidaria (Asamblea Nacional del Ecuador, 2011), lo cual es un indicador de la necesidad de dominio de un ámbito territorial en el que se aplicaría y reproduciría este modelo de organización económica, y al mismo tiempo, es una indicación de cuáles serían los sectores sociales rurales a los que se supone se direccionaría el modelo, por lo menos en forma prioritaria, al amparo de la ley mencionada. En este contexto, se intenta un ensayo evidenciado en parte de los resultados de investigaciones realizadas durante los últimos tres años sobre la sostenibilidad productiva de las Unidades Productivas Agropecuarias, UPA, en territorios ocupados por los actores de la EPS.

\section{Objetivo}

Sustentar que la posesión y uso de tierras y territorios rurales de la Sierra ecuatoriana, como escenarios funcionales para la práctica de la EPS, no es garantía para una reproducción familiar apropiada o para una vida familiar digna.

\section{Hipótesis}

La posesión y usufructo de una parcela agrícola individual o colectiva, en las áreas rurales donde prevalece la presencia de los actores de la Economía Popular y Solidaria, apenas facilita su sobrevivencia, pero no es garantía para una reproducción familiar apropiada o para una vida familiar digna.

\section{Metodología}

Entre las estrategias metodológicas aplicadas en un conjunto de investigaciones para determinar y respaldar la sostenibilidad de la producción primaria en las tierras y territorios donde se supone se encuentran los actores de la EPS, en la Sierra ecuatoriana, se destacan: el uso de las estadísticas oficiales de pobreza y desnutrición, las estadísticas de ocupación principal de la población rural y las bases de datos también oficiales sobre distribución territorial en función de la aptitud natural de uso y de su uso actual. El nivel de análisis de esta información es parroquial. La idea básica manejada es determinar y demostrar la relación de dependencia de la pobreza de la población rural con la ocupación (actividad de producción primaria) pero aplicada en tierras y territorios con aptitud de uso para conservación y/o bosques. Un enfoque metodológico complementario fue el seguimiento, caracterización y cuantificación in situ, de los tamaños de UPA y usos productivos que las familias aplican a las mismas, incluyendo la alternativa de abandono de la UPA o de parte de ella y la definición de las causas de este abandono.

\subsection{Definición de tierras y territorio, visiones sobre su tenencia y distribución}

Territorio es el entorno de inserción de la o las comunidades y es el espacio social como factor del desarrollo (Schneider \& Tartaruga, 2006). Según estos autores, se destacan tres concepciones sobre la noción de territorio: i) La concepción naturalista (territorio clásico), muy conocida y que ha justificado históricamente las guerras de conquista, a través de un imperativo funcional que se sostiene como natural, pero, en verdad es construido socialmente; ii) El territorio del individuo que pone en evidencia la territorialidad, como algo extremadamente abstracto, el espacio de las relaciones, de los sentidos, del sentimiento de pertenecer y por lo tanto, de la cultura. En este caso, el territorio toma diferentes significados, por ejemplo, para una comunidad islámica, para una tribu indígena, para una familia que vive en una gran ciudad, y iii) El concepto de espacio, o espacio geográfico, que según M. Santos, citado por Schneider \& Tartaruga (2006), sería aquel formado por un conjunto indisociable, solidario y también contradictorio, de sistemas de objetos y sistemas de acciones, no considerados aisladamente, pero como el cuadro único en el cual la historia pasa.

También sobre la definición conceptual de territorio, sin duda la concepción que se refiere a la posesión o pertenencia colectiva, es la que más prevalece y ésta es opuesta al concepto de tierra como pertenencia individual, que tiene una visión más apegada a la idea de "Propiedad plena" (Secreto, 2011). Esta autora sostiene que en las Américas, la gran diversidad sociocultural (indígenas, marrones, canoeros, pescadores, extractivistas, etc.), es acompañada de una serie paralela de diferentes usos del suelo. El reconocimiento de estas realidades por parte del Estado no implicaría necesariamente la redistribución de la tierra, sino el reconocimiento del derecho de estas comunidades a sus territorios. Entonces, la territorialidad en su dimensión de "construcción" es el esfuerzo colectivo de un grupo social por ocupar, usar, controlar e identificarse con una parte específica de su entorno biofísico, Little (2002), citado por Secreto (2011). 
Por otro lado, el concepto de "propiedad absoluta" sobre la tierra, nace en oposición a los esfuerzos colectivos de los grupos sociales por controlar sus territorios y prevalece hasta hoy, que la definición de propiedad plena, absoluta o perfecta se enfrenta con la propuesta territorial, embanderada por grupos indígenas, indígenas-campesinos, recolectores, afrodescendientes, etc. Es interesante anotar que históricamente, el concepto de propiedad plena se consolidaba en la medida que el "individualismo agrario", ha venido imponiéndose. Este fenómeno tuvo lugar, simultáneamente, en América y Europa, y que a pesar de que las realidades y experiencias sobre las cuales se superpuso la propiedad plena fueron diferentes, las teorías a partir de las cuales se legitimó esa transformación fueron las mismas (Secreto, 2011).

Se podría decir entonces, que el concepto de propiedad plena evolucionado de la noción de "individualismo agrario", habría dado lugar a lo que hoy se conoce como propiedad rural agrícola o UPA en Ecuador (de la cual un alto porcentaje todavía prevalece como propiedad sin titulación, es decir, bajo la figura de pertenencia o posesión pero no de propiedad plena); porque precisamente, ha sido un proceso evolucionado en forma sincrónica entre los intereses y reivindicaciones de las comunidades y de los campesinos no organizados, con el accionar contrapuesto de grupos de poder gestados desde la Colonia, y que más tarde (con el aparecimiento de la República), se ampararon en el poder político administrativo, ejercido desde el Estado. Como resultado de este proceso histórico de relaciones contrapuestas se evidencia la promulgación de las dos leyes de reforma agraria que han habido en el país: la Ley de reforma Agraria y Colonización de 1964 y la Ley de Reforma Agraria de 1974 y de cuya aplicación se ha logrado resolver en forma muy parcial el problema de tenencia, pero no el de distribución de la tierra. Todavía en la actualidad (junio del 2015), en Ecuador se mantiene este proceso discordante, con la construcción en camino de la Ley de Tierras y Territorios Ancestrales, en discusión en la Asamblea Nacional, que según varios analistas y líderes nacionales, en nombre de la distribución estaría consolidado la tenencia.

Por otro lado, Alimonda (2002), al referirse a la sustentabilidad de los territorios, sostiene que desde lo conceptual y como unidad de análisis, ésta tiene cuatro rasgos básicos: i) La perspectiva territorial que considera las actividades económicas; ii) La organización social en torno al ecosistema, donde la interdependencia de los actores sociales actúa como eje transversal, en la articulación territorial; iii) La perspectiva de análisis desde los actores, fundamen- talmente en situaciones de interacción, y iv) El territorio como evidencia de la manera en que la sociedad utiliza los recursos de los que dispone para la producción de bienes y la reproducción social.

Precisamente, el uso de los territorios para la producción de bienes y el aseguramiento de la reproducción social sustentada por (Alimonda 2002), parecería estar en riesgo con el apogeo del modernismo globalizante en que vivimos, que presiona por el uso múltiple de los territorios, que muchas veces pospone el uso primordial como es la producción de bienes primarios (alimentos, fibras, forrajes y otros), para dar paso a usos extractivos de recursos no renovables, con una carga inmanejable de pasivos ambientales que mina inexorablemente la capacidad y el potencial de los territorios para producir bienes primarios. Esta tendencia es muy bien sustentada por Stella (2008), quien define el tema de la siguiente manera "...en el contexto actual de la globalización donde todo se "mercantiliza", el espacio rural deja de ser considerado el sustento de la producción de alimentos y se reconocen en él múltiples actividades..."; dando paso a que el territorio pierda su calidad de instrumento para la sustentabilidad de la población.

Curiosamente, esta tendencia es alimentada por muchos gobiernos, que imbuidos por la necesidad de extraer recursos no renovables, fomentan usos contrarios a la conservación y uso sustentable de las tierras y territorios. Y, aunque resulte paradójico, salvo algunas excepciones, se observa que también las comunidades rurales e incluso los grupos étnicos ancestrales que empujados o no por la "modernidad", dejan de mirar a su territorio como fuente de sustento desde su capacidad productiva de bienes (principalmente alimentos) y empiezan a valorar otras posibilidades incluyendo la extracción de recursos no renovables, dentro de la lógica de "múltiples usos", como alternativa a la sola posibilidad de producir bienes primarios.

\section{Resultados y discusión}

\subsection{La calidad de las tierras o territorios como es- cenarios de la Economía Popular y Solidaria}

Si un objetivo de la Ley de EPS es "Potenciar las prácticas que se desarrollan en las comunas, comunidades, pueblos y nacionalidades, y en sus unidades económicas productivas para alcanzar el Sumak Kawsay", es preocupante que precisamente 
en los territorios ocupados por estos sectores de la población, es en donde se observan las limitaciones más abultadas, que obstaculizan la pretendida potenciación de sus prácticas productivas.

En primer lugar, la gran mayoría de los sectores de la población rural que serían los actores de la EPS, están localizados (ocupan territorios) de lo más marginales y con las mayores limitaciones para una actividad productiva; es decir, son territorios o tierras cuya aptitud natural no es compatible con la actividad productiva (actividad agropecuaria o de producción primaria). En segundo lugar, son estos territorios, que precisamente por su condición de marginalidad están sujetos a riesgos en forma permanente. Riesgos a eventos adversos de orden climático, totalmente no controlables, por los actores. Estos riesgos están evidenciados por las amenazas constantes de fenómenos climáticos adversos como: nevadas, heladas, granizadas, sequías, inundaciones, vientos, etc.; en combinación con la vulnerabilidad de los territorios, precisamente por estar ubicados en ecosistemas marginales para funciones productivas.

Esta situación de riesgo constante al que están sometidos los territorios marginales mencionados, evidentemente tiende a agravarse como consecuencia de las secuelas del cambio climático; así, según un último informe del Programa Mundial de Alimentos (PMA, 2014), en Ecuador hay 389 parroquias que sufren de grave vulnerabilidad y 586 de muy alta vulnerabilidad y el cambio climático está incrementando la frecuencia e intensidad de los desastres naturales como: sequías, inundaciones, granizadas, tormentas de nieve y heladas, entre otros.

Uno de los indicadores idóneos de la capacidad productiva de un espacio territorial es el conocido como "Aptitud natural de uso", entendida como "la vocación de la tierra para un uso específico, es decir, el nivel de adecuación del terreno considerando sus características naturales" ("Evaluación de tierras para la agricultura de regadío", 1985). Una vez definida la aptitud natural de uso de un territorio determinado, se espera que los actores sean consecuentes con ésta y apliquen funciones productivas en armonía con la capacidad natural de uso del suelo o se espera la existencia de una política pública para conseguir esta armonía; sin embargo, se ha encontrado que en Ecuador, la mayoría de los tenedores de tierra, incluyendo las comunas, comunidades, pueblos y nacionalidades (aparentemente presionados por la necesidad de generar ingresos), desconocen y trasgreden esta característica y aplican funciones productivas totalmente incompatibles con la aptitud natural de uso de los territorios que ocupan.

En un intento por corroborar las observaciones sobre la transgresión de la aptitud natural de uso del suelo, como parte de las investigaciones sobre la sostenibilidad de los sistemas productivos de las UPA, en territorios de comunidades rurales en la Sierra ecuatoriana, se determinó la relación o interacción entre la pobreza de las poblaciones, con los porcentajes de esas poblaciones que se dedican a producción primaria, y a su vez, con los indicadores de aptitud natural de uso de los territorios que ocupan esas poblaciones, expresados en: i) El porcentaje del territorio con aptitud para producción agrícola, y ii) El porcentaje del territorio con aptitud para bosques y/o conservación. Es decir, se intentó definir, cómo la pobreza de las poblaciones depende o se relaciona con la actividad productiva y con la aptitud natural de uso de los territorios que ocupan. Se trabajó con los territorios y poblaciones de las parroquias rurales de las tres provincias del norte de la Sierra ecuatoriana: Carchi, Imbabura y Pichincha. Parte de la investigación en las provincias de Imbabura y Pichincha fue desarrollada como disertación de grado de dos egresadas de la Escuela de Ciencias Geográficas (Nieto, 2014).

En las Figuras 1 a 3, se presentan, para los dos quintiles de las parroquias rurales, cuyos territorios tienen mayoritariamente aptitud natural de uso para bosques y conservación, en las tres provincias estudiadas, los patrones de comportamiento, con una relación de correspondencia directa entre los porcentajes de pobreza de las poblaciones parroquiales y los porcentajes de territorios con aptitud para conservación y/o bosques. También se observa una correspondencia directa entre estas dos variables con los porcentajes de la población dedicados a agricultura como actividad principal generadora de ingresos.

Si la actividad principal generadora de ingresos de las comunidades es la agropecuaria y si ésta se hace en territorios no aptos para esta actividad, entonces es de esperarse que los ingresos netos por su actividad sean bajos, nulos y hasta negativos y por lo tanto, es incuestionable que el primer factor de pobreza de las comunidades es el ingreso neto bajo o nulo, por sus actividades productivas. Muchos productores de las áreas rurales estudiadas, 
se mantienen ocupados en estas actividades primarias, algunas de carácter extremadamente deprimidas, aun sabiendo de sus bajas o nulas posibilidades de ingresos netos, simplemente por el fenómeno de "Costo de oportunidad de la mano de obra", que para esas condiciones es cero o cercano a cero (Nieto, 2011; Nieto \& Caicedo, 2012) y, mientras no aparezca una oferta de ocupación de mano de obra en sus ámbitos locales, las opciones que les queda son: continuar con estas actividades de ingreso deprimido o la emigración, cosa que es evidente, por lo menos para un gran sector de la PEA rural de Ecuador.

Cabe destacar o explicar algunas aparentes excepciones, que se pueden apreciar en las figuras mencionadas, las que en realidad confirman la observación y no son la excepción como aparecen. Por ejemplo, en la Figura 3, se aprecia que para la provincia de Pichincha, los niveles de pobreza son visi- blemente más bajos que en los casos de las provincias de Imbabura y Carchi, lo cual, evidentemente, es la consecuencia de las mayores y mejores oportunidades para generar ingresos que ofrece esta provincia, por la influencia del Distrito Metropolitano de Quito, DMQ, hacia donde las familias, especialmente los miembros de la PEA, emigran desde las parroquias rurales en forma temporal o intermitente, (incluso hay un flujo diario en horas laborables hacia el DMQ, especialmente desde las parroquias más cercanas o circundantes), en busca de actividades ocupacionales generadoras de ingresos. Esta misma circunstancia hace que también los porcentajes de la población rural de esta provincia, dedicada a actividades agrícolas sean menores, comparados con los de las otras dos provincias en estudio, aspecto sobre el cual sobresale la parroquia Mindo, que tiene una oferta turística reconocida, de la cual se ocupa y vive mayoritariamente la población.

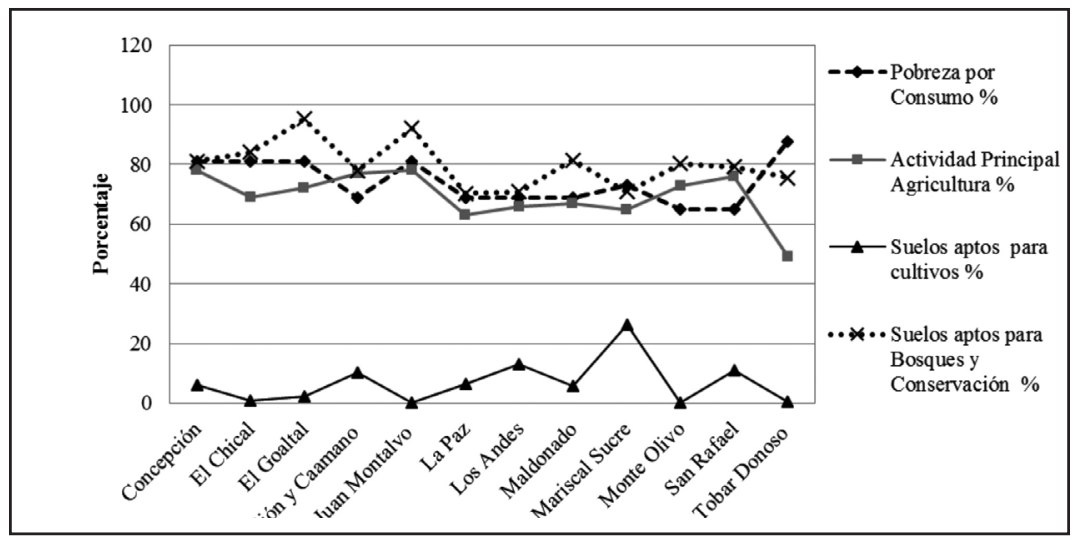

Figura 1. Relaciones entre Pobreza de la población, porcentaje de la población con actividad principal "Agricultura" y aptitud productiva de los territorios, para 12 parroquias rurales de la provincia de Carchi.

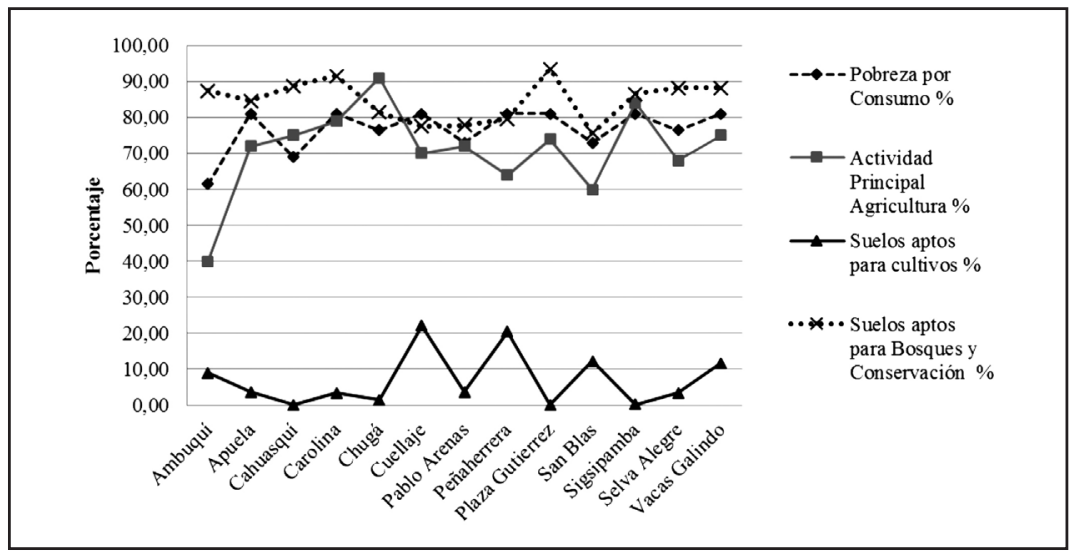

Figura 2. Relaciones entre Pobreza de la población, porcentaje de la población con actividad principal "Agricultura" y aptitud productiva de los territorios, para 13 parroquias rurales de la provincia de Imbabura. Tomado y adaptado de Nieto (2014). 


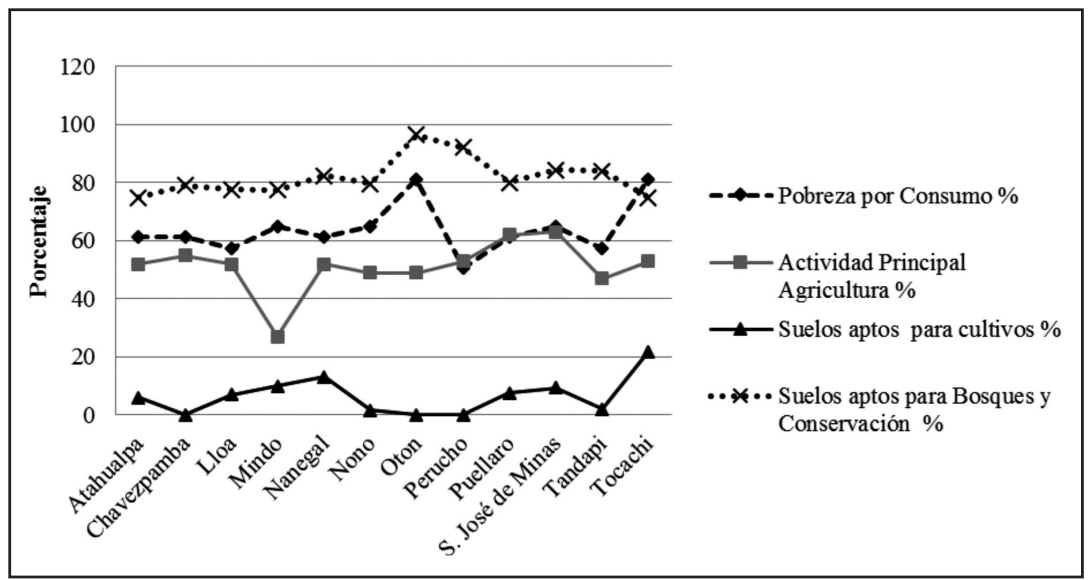

Figura 3. Relaciones entre Pobreza de la población, porcentaje de la población con actividad principal "Agricultura" y aptitud productiva de los territorios, para 12 parroquias rurales de la provincia de Pichincha. Tomado y adaptado de Nieto (2014).

En las Figuras 1, 2 y 3, también se representan los porcentajes de los territorios parroquiales con aptitud para actividades agrícolas. Es evidente, que en forma consistente, para las parroquias analizadas, en las tres provincias, los porcentajes de sus territorios con esta aptitud no superan el $20 \%$. Entonces, se explican los niveles de pobreza de la población de las parroquias rurales por cuanto su actividad mayoritaria generadora de ingresos es la agropecuaria, pero los porcentajes de sus territorios aptos para esta actividad son tan bajos que no permiten que las familias dedicadas a esta actividad obtengan los ingresos netos esperados.

Varios autores en la literatura confirman la relación directa entre la pobreza de los grupos rurales y su actividad primaria relacionada con producción agropecuaria (Moreno, 2008; Banco Mundial, 2004; Chiriboga \& Wallis, 2010; Larrea et al., 2008), pero no señalan la relación directa entre actividad de producción primaria y la aptitud de los suelos en donde realizan esta actividad. Así, según un informe del Banco Mundial del 2004, el 40\% de la población de Ecuador vive en las zonas rurales. Dos tercios de esa población son pobres y una elevada proporción de éstos son extremadamente pobres y se concluye que los ingresos bajos de los hogares pobres de las zonas rurales suelen estar atados a los en extremo bajos niveles de producción de los agricultores; mientras que para Chiriboga \& Wallis (2010), el mayor número de pobres se encuentra entre las unidades de agricultura familiar o de subsistencia y trabajadores agrícolas eventuales sin tierra, de los cuales, los primeros parecen localizarse sobre todo en la Costa, mientras los segundos en la Sierra y Amazonia. Además, agregan que hay una ausencia de información suficiente sobre las dinámicas de pobreza vinculadas a los trabajadores agrícolas que debería investigarse; esto incluye conocer mejor los mercados laborales agrícolas y sus necesidades, como también los mercados financieros rurales. Estos autores insisten en que las posibles soluciones irían por el lado de las propuestas de innovación en el medio rural y que tiene que ver con la forma como dichas unidades se articulan a servicios de crédito, asistencia técnica, capacitación y otros. Sin embargo, es evidente que estas propuestas, hasta ahora no han funcionado y más bien, han coadyuvado a cimentar la pobreza entre los actores, precisamente porque tanto los investigadores como los tomadores de decisiones han ignorado los problemas estructurales más profundos como la calidad y cantidad de los medios de producción disponibles, principalmente tierra agrícola.

Por su parte Larrea et al. (2008), reconocen que en general, la pobreza es mayor en las áreas rurales, y afecta principalmente a las zonas altas de la Sierra central, con elevada población indígena, así como a la Amazonía, y a ciertas regiones de la Costa con alta presencia campesina y de asalariados agrícolas. Reconocen además, que los cambios ocurridos en la última década han beneficiado principalmente a Quito, a las ciudades intermedias de la Sierra, a Guayaquil, y a regiones rurales muy definidas con agricultura empresarial moderna intensiva, pero tampoco se preocupan de la calidad de los medios de producción disponibles en los sectores rurales más pobres. 
Estos autores Larrea et al. (2008), mencionan que las parroquias que más han reducido su pobreza, han sido aquellas con mayor proporción de emigración internacional. Estas parroquias corresponden en general a zonas deprimidas, pero no incluyen las áreas más pobres del país, como algunas parroquias con población indígena mayoritaria, donde la emigración internacional es menos importante, debido a que la carencia de recursos y los bajos niveles de educación merman la viabilidad de la migración internacional, que requiere una considerable inversión inicial. Además, reconocen que los indígenas tienen resistencias a la emigración internacional por su apego cultural a la tierra y a sus lugares de origen.

En consecuencia, a la luz de los resultados de la investigación que confirman la relación entre pobreza de la población con su actividad en producción primaria y con la aptitud productiva de sus tierras; resultados que son corroborados con las opiniones de los investigadores citados, cabe una reflexión, el reconocimiento y asentimiento del uso productivo de los territorios rurales, en producción de bienes primarios (por ejemplo, los necesarios para sustentar el modelo de la EPS), se vuelve insostenible. Y entonces, si esto es así, ¿cómo se debe entender el interés político de sostener a estos grupos en estos territorios fungiendo de productores primarios? Acaso se debe suponer la existencia de un sector de la población (las comunidades y colectivos rurales marginales), para los cuales el Sumak Kawsay es la resignación y aceptación de continuar como tradicionalmente han hecho, apegados a la tierra o a su territorio, produciendo abnegadamente bienes primarios en condiciones cada vez menos ventajosas, que les desmejora su calidad de vida; para facilitar el flujo de bienes en una forma unidireccional (campo-ciudad). Y todo esto, para atender a otro grupo de la población (los urbanos) que entiende el Sumak Kawsay, como la oportunidad para exigir cada vez mejores condiciones de vida. Lo más preocupante es que históricamente las políticas públicas han sido para alentar este modelo de flujo unidireccional de recursos desde el campo hacia la ciudad. Esto es ratificado por Rosero, Carbonel \& Regalado (2011), quienes presentan un análisis histórico que evidencia cómo las políticas públicas nacionales emanadas de los diferentes gobiernos de turno, durante los últimos 40 años, han favorecido directa o indirectamente el traslado de recursos (subsidios) desde los campos hacia las ciudades.

\subsection{La tierra un recurso escaso para los actores de la Economía Popular y Solidaria}

Para completar el cuadro de factores que obstaculizan la potenciación de las UPA, como medio de desarrollo y de reproducción social (sin importar el modelo económico productivo a aplicar), se destaca la escasez del recurso tierra o territorio, es decir, los tamaños de parcela disponibles (parcela individual o colectiva), no alcanzan los tamaños mínimos necesarios para una actividad redituable. La limitación del tamaño de parcela para una actividad rentable, conocido como mini o micro fundo, es especialmente evidente en las regiones Sierra y Costa, y aunque por el momento parece ser menos evidente en la Amazonia, la tendencia a la división de la UPA, es la misma que en las otras dos regiones (Nieto \& Caicedo, 2012). La partición de la tierra hasta llegar a tamaños de parcela insignificantes para una producción primaria mínima rentable se da por vía de la herencia o simplemente por venta y traspaso de una parte de la propiedad; fenómeno que es creciente y aparentemente irreversible. En algunos casos la división de la tierra, ha llegado a límites tales que la parcela apenas alcanza para un espacio de vivienda familiar, lo cual convierte a los campesinos en meros habitantes rurales, cuya dependencia económica es de fuentes de ingresos totalmente externas a la gestión de la UPA, aunque en las estadísticas nacionales siguen constando como agricultores.

En este contexto, la tierra como pertenencia individual, más bien se ha convertido en el principal y acaso único activo del patrimonio familiar, antes de ser el medio de producción, y en cuanto a los territorios como pertenencia colectiva, en muchos casos, también siguen el mismo camino, aunque antes hay que vencer las dificultades de pasar de propiedad colectiva a propiedad individual, mediante la parcelación equitativa del territorio entre los miembros del colectivo. En Ecuador, esta tendencia parece irreversible, a no ser que se imponga una política pública que prohíba, la partición de la UPA individual o colectiva, precisamente para asegurar el uso de la tierra en la producción de bienes primarios, pero principalmente en la producción de alimentos, para garantizar la seguridad alimentaria de la población. Esta proposición es válida para sustentar el modelo de EPS como para sustentar cualquier otro modelo económico.

En el caso de Ecuador, desde la Reforma Agraria en 1964, la disponibilidad de tierra cultivable ha 
sufrido un recorte muy significativo. Así, el $2.5 \%$ de los productores en Ecuador poseen menos de 0,38 hectáreas y el $63.5 \%$ de los productores dispondrían de apenas 1.4 hectáreas, en promedio. Estas son superficies de tierra irrisorias para poder "levantar el edificio campesino" en base únicamente a las actividades agropecuarias (Martínez, 2004). Además de la escasez de tierra está la inseguridad en la tenencia, por no tener títulos de propiedad para la tierra individual y colectiva de una gran parte de los campesinos e indígenas del Ecuador (Nieto, 2004; Martínez, 2004), lo cual les limita el acceso a varios beneficios, pero principalmente al crédito para hacer inversiones en sus unidades productivas e incluso les restrinje el acceso a los beneficios "subsidios" que en ocasiones se han ofrecido desde los gobiernos de turno, para incentivar la producción agropecuaria o para mejorar la seguridad alimentaria de las propias familias beneficiarias.

En la Tabla 1, se presenta un resumen de la información oficial disponible con respecto a la distribución de la tierra de cultivo en Ecuador, por número y porcentaje de las UPA, de acuerdo a los rangos de tamaño de las mismas. Efectivamente, el porcentaje de Unidades Productivas cuyo tamaño es inferior a una hectárea es $29.5 \%$ del total y, sumado éste al conjunto con tamaños entre 1 y 5 hectáreas, se llega al $63.51 \%$ de las mismas, aunque el porcentaje de tierra que controlan es apenas $6.27 \%$. Esto significa que más del $63 \%$ de las familias (es decir algo más de 535,900 unidades productivas, de las 843,882 existentes a nivel nacional), y que constan en las estadísticas oficiales como "agricultores" en Ecuador, en realidad no lo son, porque la gestión o cultivo de estas micro o mini unidades productivas no les proporciona ingresos para garantizar una vida digna de sus familias. Considerando que esta información proviene del Censo Agropecuario Nacional del 2000, es de suponer que en la actualidad (2015), la proporción de mini o micro fundos con relación al total, será sustancialmente superior, puesto que durante este periodo, no ha habido ninguna política nacional ni ley que impida o desvíe la tendencia a dividir los tamaños de UPA.

Tabla 1. Distribución de la tierra cultivada en Ecuador por número y porcentaje de UPA, de acuerdo a los tamaños de parcela, con base en el Censo Agropecuario 2000.

\begin{tabular}{lccccccc}
\hline Tierra/UPA & \multicolumn{7}{c}{ Tamaños de UPA (ha) } \\
\cline { 2 - 8 } & $<\mathbf{1}$ & $\mathbf{1 ~ a ~ 5}$ & $\mathbf{5}$ a 10 & $\mathbf{1 0 ~ a ~ 5 0}$ & $\mathbf{5 0 ~ a ~ 1 0 0}$ & $>\mathbf{1 0 0}$ & Total \\
\hline Total superficie & 95,835 & 678,391 & 688,987 & $3,389,834$ & $2,242,409$ & $5,260,375$ & $12,355,831$ \\
Porcentaje & 0.8 & 5.47 & 5.6 & 27.4 & 18.15 & 42.57 & 100 \\
Total UPA & 248,398 & 286,911 & 101,066 & 152,452 & 34,498 & 19,557 & 842,882 \\
Porcentaje & 29.5 & 34.01 & 11.98 & 18.1 & 4.09 & 2.32 & 100 \\
\hline
\end{tabular}

Fuente: INEC, Censo Nacional-2010; Larrea Maldonado (2008); Carrión \& Herrera (2012)

El tamaño de UPA, por debajo del mínimo necesario para obtener ingresos apropiados y excedentes, que permitan la sobrevivencia digna de las familias rurales, es quizá la mayor dificultad que enfrenta hoy el Ecuador. Aunque para muchos investigadores la solución es una nueva reforma agraria, para afectar los "fundos grandes en pocas manos" y disminuir la inequidad en la tenencia de la tierra, mediante el reparto de tierras a las familias con escasa o ninguna tierra (Rosero, Carbonel \& Regalado, 2011; Isch, 2010; Lefeber, 2008; Carrión \& Herrera, 2012); probablemente para Ecuador no es conveniente otra reforma agraria, en términos únicamente de reparto de tierras. Modernamente un proceso de reforma agraria, además de la redistribución de las tierras, debería considerar temas complementarios que son elementos clave para elevar la productividad y la competitividad de la actividad agropecuaria, así: el respeto a la aptitud natural de uso del suelo, para repartir o distribuir solamente tierras cultivables; la tecnificación de los sistemas productivos, para optimizar el uso de recursos escasos; la gestión de cadenas productivas integrales, que permitan la participación de los productores en actividades de generación de valor agregado a su producción; la profesionalización y la dignificación de la ocupación de agricultor, para entender que es una ac- 
tividad digna y respetable, y para evitar el reparto de tierras a ciudadanos sin vocación o apego a la actividad agropecuaria y que solo pretenderían acceder a la tierra para crear o incrementar el patrimonio familiar. Pero, por sobre todo, habría que considerar la redistribución de tierras en parcelas del tamaño mínimo para lograr una gestión rentable y bajo un condicionamiento legal capaz de impedir la partición posterior de la parcela. Para corroborar las condiciones en las que se desenvuelven las unidades productivas en las comunidades que son escenarios de la EPS, se presenta una parte de los resultados de las investigaciones realizadas en varias comunidades de la Sierra, sobre la aplicación de indicadores de sostenibilidad del fun- cionamiento de estas unidades productivas. Es el caso de dos comunidades Santa Rosa y Porotog, de la parroquia Cangahua, cantón Cayambe, en la provincia de Pichincha, donde se trabajó con el 10\% de las UPA (familias), de cada comunidad, tomadas al azar. El indicador estudiado fue la disponibilidad de la tierra o tamaño de la UPA y el uso de la misma. Se encontró una diferencia muy notoria en cuanto al tamaño de UPA por comunidad; así, el promedio de tamaño de UPA para la comunidad de Santa Rosa fue de apenas 0,81 ha, con un máximo de 2.5 ha y un mínimo de 0.1 ha; mientras que el promedio para la comunidad de Porotog es de 4.89 ha por UPA, con un máximo de 7.0 ha y un mínimo de 3.5 ha (Figura 4 ).

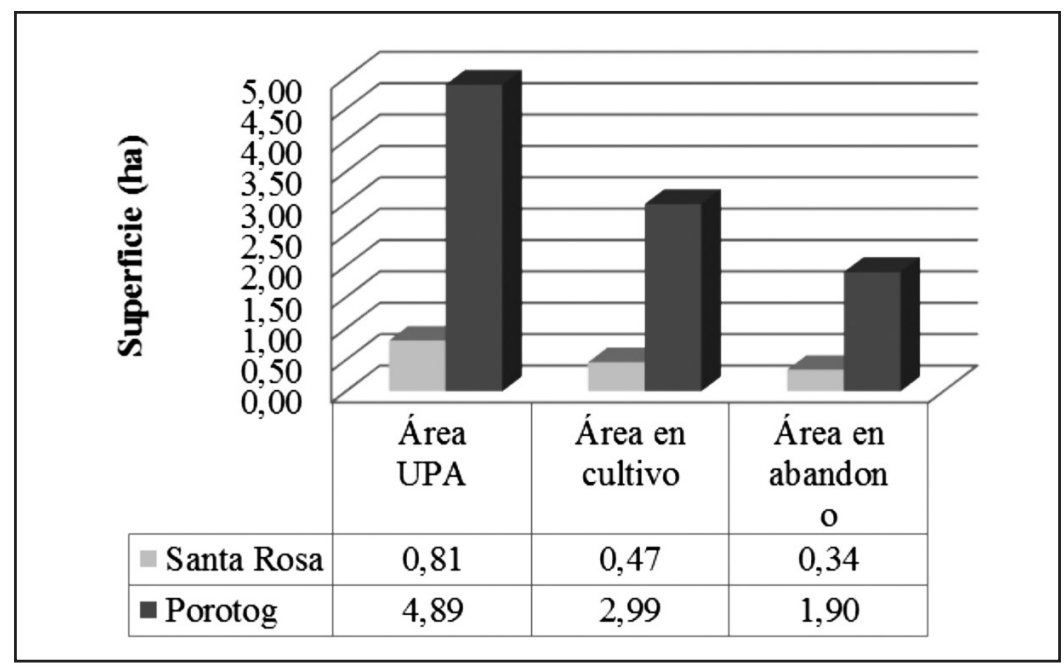

Figura 4. Distribución de los promedios de área por UPA y las proporciones puestas en cultivo y en abandono, para las comunidades Santa Rosa y Porotog, parroquia Cangahua, cantón Cayambe.

A pesar de estas diferencias en favor de los tamaños de UPA de la comunidad de Porotog, estos datos son evidencia clara de la existencia de mini y micro fundos en ambas comunidades estudiadas. Sin embargo, en algunos casos, se encontró que las familias no son capaces de poner en cultivo el 100\% del área disponible, así: en la comuna Santa Rosa, en promedio se deja sin cultivar cerca del $43 \%$ de la UPA, con una superficie promedio ocupada en cultivos y pasturas de 0.47 ha, y una superficie promedio en estado de abandono de 0.34 ha. Del mismo modo, en la comuna Porotog, se abandona cerca del 39\% de la superficie de la UPA, con 2.99 ha cultivadas y 1.9 ha sin cultivar, en promedio por UPA.

Esta figura de abandono, sin cultivar, de porcentajes significativos de las unidades productivas, a pesar del minifundio existente, se repite sistemáticamente en otras comunidades de la Sierra, donde se está realizan- do la misma investigación y es un aspecto para reflexionar, porque es un fenómeno que parecería no sustentar la afirmación generalizada de que el subdesarrollo y la pobreza de las familias rurales es por falta de tierra cultivable y que por lo tanto, una solución es afianzar una nueva reforma agraria para redistribuir la tierra en su favor. Como elementos para entender el abandono de parte de las unidades productivas, se ha podido evidenciar los siguientes: i) La falta de competitividad de la agricultura ecuatoriana en general, debido a la elevación de los costos de producción, especialmente en los rubros mano de obra e insumos extra finca; ii) la exagerada partición de la UPA, que al llegar a tamaños extremadamente pequeños, como los observados en la comunidad de Santa Rosa, están muy por debajo de los tamaños mínimos requeridos para hacer una agricultura rentable y por lo tanto, las familias pierden el interés en cultivarlas; iii) La extrema disminución y hasta pérdida total de la fertilidad de los suelos, debido 
no solamente a la aplicación sistemática de prácticas contrarias a la conservación, sino a que los suelos desde su condición natural original no tienen aptitud agrícola; iv) Las características agroecológicas de los agro ecosistemas de la mayoría de las UPA no empatan con la aptitud de uso agrícola, más bien son de aptitud forestal o para conservación, y v) La dificultad de mecanizar por lo menos algunas de las actividades de cultivo, ya sea por la topografía irregular, por su tamaño pequeño o por la falta de herramientas mecánicas apropiadas.

Muchas de las actividades rurales, por la dureza y esfuerzo que demandan a cambio del muy poco ingreso que se obtiene, no son atractivas para la mano de obra rural. Quizá lo que falta entender y aceptar es que el mundo moderno está poniendo otros valores y "oportunidades" para el ser humano, dejando el trabajo duro para la máquina y este mensaje modernizante también está llegando al área rural ecuatoriana, gracias al milagro de la comunicación y de la informática. En estas condiciones, se evidencia que el tipo de agricultura denominada "agricultura familiar", "agricultura de subsistencia", o "agricultura de sobrevivencia", la cual es todavía reivindicada por varios autores (Isch, 2010; Rosero, Carbonel \& Regalado, 2011; Carrión \& Herrera, 2012), parecería no tener futuro. Es hora de empezar a reflexionar muy en serio sobre el hecho de que el desarrollo rural no tiene futuro solamente con actividades productivas primarias.

Otro indicador estudiado fue la distribución de la porción cultivada por UPA, en los dos usos principales: agricultura y plantación de pasturas para crianza de animales. En este caso, debido a que la crianza de animales, especialmente bovinos, requiere de superficies considerables de tierra para poner en pasturas, se encontró que en la comunidad de Porotog, donde la superficie de tierra por UPA es superior, se dedica más área a la producción de pasturas, -más de 2 hectáreas en promedio-, comparando con apenas 0.85 ha dedicadas para cultivos (Figura 5), lo que significa algo más del $71 \%$ del área de la UPA en promedio dedicada a pasturas y apenas el $29 \%$ con dedicación a cultivos agrícolas. Sin embargo, también en la comunidad Santa Rosa, la tendencia a utilizar parte de la UPA en la plantación de pasturas para la cría de animales, es significativa, con casi un $28 \%$ ( $0.13 \mathrm{ha})$, dejando el $72 \%$ (0.33 ha) en cultivos.

La tendencia a cambiar el uso del suelo de sistemas agrícolas a sistemas pecuarios, es otro fenómeno generalizado en toda la Sierra ecuatoriana y entre las razones que explican la tendencia están las siguientes: i) La escasez de mano de obra, para atender cultivos que son altamente demandantes en labores manuales, mientras que las actividades ganaderas, son relativamente menos exigentes en mano de obra; ii) La mayoría de labores de atención a los pequeños sistemas pecuarios pueden ser atendidos por niños o adultos mayores; mientras que las actividades agrícolas requieren de mano de obra especializada o de mayor desgaste de energía, debido al esfuerzo requerido; iii) La relativa poca exigencia de insumos (especialmente agroquímicos) por parte de los sistemas pecuarios, en comparación con los sistemas agrícolas, y iv) La relativa estabilidad de precios y seguridad de mercado para productos derivados de la ganadería, especialmente leche, frente a la alta inestabilidad de precios de los productos agrícolas.

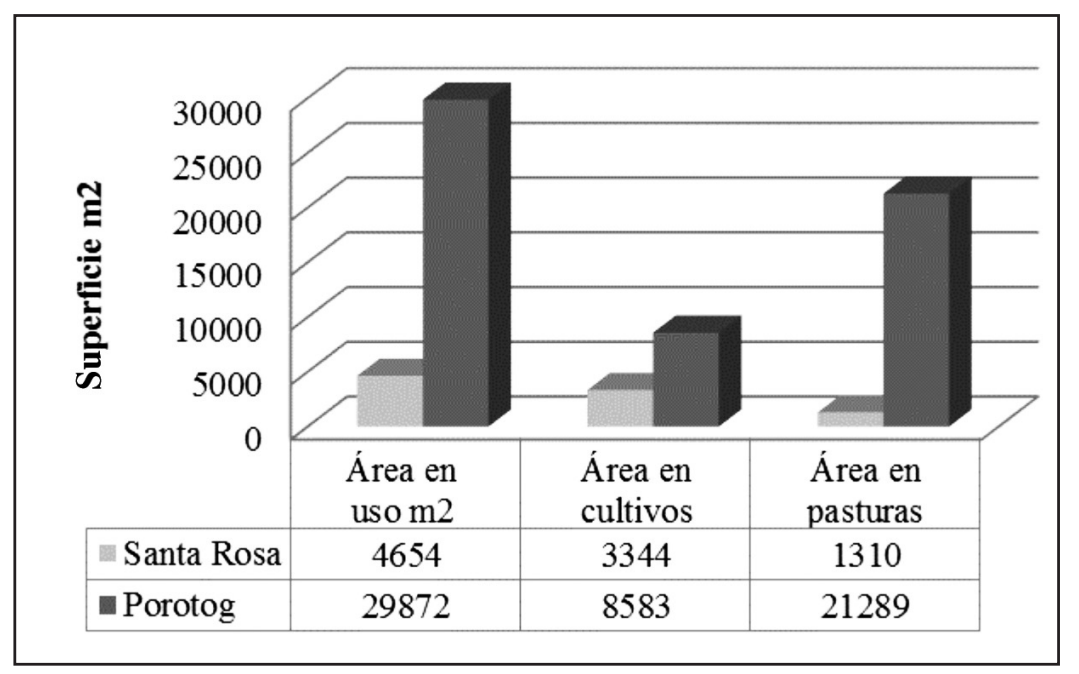

Figura 5. Distribución de los promedios de área $\left(\mathrm{m}^{2}\right)$, puesta en cultivo por UPA y las proporciones de área en agricultura y en pasturas, para las comunidades Santa Rosa y Porotog, parroquia Cangahua. 
Entonces, de estos resultados y su análisis, se empieza a vislumbrar la realidad de la situación de las familias rurales, cuyo sustento deja o ha dejado de depender de las actividades de laboreo del campo como se ha creído y aún se cree, y por lo tanto se empieza a encontrar evidencias de que el "funcionamiento de las UPA, dentro de la categoría producción familiar o de subsistencia no tiene sostenibilidad, en términos económicos". Aparentemente, ignorando estas realidades para el área rural, tradicionalmente, gran parte de los programas de extensión y desarrollo rural y en general la política gubernamental (incluyendo la política del Gobierno actual), han sido encaminados al fomento de la producción de alimentos de consumo nacional, especialmente con los pequeños agricultores; pero seguramente los intereses y las conveniencias de éstos son contrarios, por las razones expuestas. $Y$ esto evidentemente tendría una repercusión en el funcionamiento del modelo de EPS, o por lo menos minaría las posibilidades de su consolidación.

\section{Conclusiones}

Tanto por la calidad de la tierra o territorio (aptitud natural de uso), como por la cantidad disponible (tamaño de UPA), la posesión y usufructo de una UPA, no es una garantía para la reproducción $\mathrm{y}$ aseguramiento de una vida familiar, aun bajo el modelo de EPS, en la mayoría de áreas rurales ecuatorianas, pero especialmente para las comunidades pueblos y nacionalidades asentadas en ecosistemas marginales.

Son estos territorios, que precisamente por su condición de marginalidad están sujetos a grandes riesgos ya que presentan las mayores vulnerabilida-

\section{Referencias}

Alimonda, H. (2002). Ecología política. Naturaleza, sociedad y utopía. Buenos Aires: CLACSO.

Banco Mundial. (2004). Ecuador: Evaluación de la pobreza, unidad sectorial de reducción de la pobreza y gestión económica en America Latina y el Caribe. Washington D.C.: Banco Mundial. des. Los riesgos están en función de las amenazas a fenómenos climáticos adversos como: nevadas, heladas, granizadas, sequías, inundaciones, vientos destructivos, etc.; y la vulnerabilidad se evidencia, precisamente por la ubicación en ecosistemas marginales para funciones productivas. Esta situación de riesgo, tiende a agravarse con las secuelas del cambio climático.

El uso agropecuario, incompatible con la aptitud natural de uso de la tierra es una de las principales causas de la pobreza rural, lo que es más evidente en los territorios ocupados por etnias ancestrales como lo demuestran las estadísticas de pobreza y desnutrición.

La división de la UPA, ha llegado a límites tales que en algunos casos la parcela apenas alcanza para un espacio de vivienda familiar, lo que significa que gran parte o la mayoría de los campesinos se han convertido en habitantes rurales, cuya dependencia económica es de fuentes externas a la gestión de la UPA, aunque en las estadísticas nacionales siguen constando como agricultores.

En consecuencia, en muchos casos, la tierra como pertenencia individual o colectiva, más bien se ha convertido en el principal y acaso único activo del patrimonio familiar o comunal, antes de ser el medio de producción.

Se ha evidenciado que el tipo de agricultura denominada "agricultura familiar", "agricultura de subsistencia", o "agricultura de sobrevivencia", la cual es todavía reivindicada por varios autores, incluyendo los promotores del modelo EPS, parecería no tener futuro promisorio, más todavía, cuando la tendencia a subdividir las UPA, es creciente.

Carrión D. \& Herrera, S. (2012). Ecuador rural del siglo XXI. Soberanía alimentaria, inversión pública y politica agraria. Quito: Instituto de Estudios Ecuatorianos.

Castillo Gordón, N. \& Vaca Navarrete, N. (2014). Influencia de la aptitud natural del suelo y uso del suelo en relación con la probreza y desnutrición de los territorios 
rurales en las provincias de Pichincha e Imbabura. PUCE, Ciencias Geográficas. Quito, Ecuador: PUCE.

Chiriboga, M. \& Walis, B. (2010). Diagnóstico de la pobreza rural en Ecuador y respuestas de política pública. Grupo de Trabajo sobre pobreza rural.

Coraggio, J. L. (sf). La economía popular solidaria en el Ecuador. Argentina: Instituto del Conurbano de la Universidad Nacional de General Sarmiento.

Asamblea Nacional del Ecuador. (2011). Ley Orgánica de Economía Popular y Solidaria del Sistema Financiero. Quito: Asamblea Nacional. Registro Oficial 444.

FAO (1985). Evaluación de tierras para la agricultura de regadío. Boletín de Suelos 55.

Isch, E. (2010). La acumulación en el agro: una realidad presente que no queremos en el futuro. En SIPAE, Tierra y Agua: Interrelaciones de un acceso inequitativo. Quito, Ecuador: SIPAE.

Larrea, C.; Landín, R.; Larrea, I.; Werborich, W. \& Fraga, R. (2008). Mapas de pobreza, consumo por habitante y desigualdad social en Ecuador: 1995-1996 (Vol. Documento de trabajo número 13). Santiago, Chile: RIMISP.

Larrea Maldonado, C. (2008). Tenencia de la tierra, cambios agrarios y etnicidad indígena en el Ecuador: 1954-2000. En N. L. John, Desarrollo rural y neoliberalismo, Ecuador desde una perspectiva competitiva. Quito: Universidad Andina Simón Bolívar, Sede Ecuador. Corporación Editorial Nacional.

Lefeber, L. (2008). La agricultura y el desarrollo rural. Una crítica a las políticas de establishment en Ecuador. En L. North \& D. C. Editores, Desarrollo rural y neoliberalismo. Ecuador desde una perspectiva comparativa. Quito: Universidad Andina Simón Bolívar, Sede Ecuador. Corporación Editora Nacional.

Maris Shmite, S. (2008). Territorio y sustentabilidad: El "Caldenal" en la lógica actual de capitalismo. Argentina: Departamento de Geografía. Facultad de Ciencias Humanas. Universidad de la Pampa.

Martínez, L. (2004). El campesino andino y la globalización a fines de siglo (una mirada sobre el caso ecuatoriano). Revista Europea de Estudios Latinoamericanos y del Caribe 77.
Moreno, J. (2008). Hacia una historia social de la pobreza en la Audiencia de Quito. Una revista de historia social y literatura de América Latina.

Nieto, C. (2014). Influencia de la aptitud natural del suelo y uso del suelo en relación con la pobreza y desnutrición de los territorios rurales en las provincias de Pichincha e Imbabura. Quito: PUCE.

Nieto C. (2011). El Salario Digno del agricultor ecuatoriano: acceso, limitaciones sociales y financieras. GEOPUCE. Revista de la Escuela de Ciencias Geográficas, 3, 87-94.

Nieto C. (2004). El acceso legal a la tierra y el desarrollo de las comunidades indígenas y afro-ecuatorianas: La experiencia del PRODEPINE en Ecuador. Revista Reforma Agraria Colonización y Cooperativas, FAO, (2004/1), 97-109.

Nieto C. \& Caicedo C. (2012). Análisis reflexivo sobre el desarrollo agropecuario sostenible, en la Amazonía ecuatoriana. Joya de los Sachas, Ecuador: EECA, INIAP.

PMA. (27 de abril de 2014). Ambiente: Áreas vulnerables por el cambio climático se muestran en un atlas. El Comercio, Quito, Ecuador, pág. 19.

Remigio y Sánchez. (2004). Estado y gestión de los recursos hídricos en el Ecuador.

Rosero, F. Carbonel, Y. \& Regalado F. (2011). Soberanía alimentaria, modelos de desarrollo y tierras en Ecuador. Quito, Ecuador: CAFOLIS, Grupo de apoyo.

Schneider S. \& Tartaruga, G. (2006). Territorio y enfoque territorial: De las referencias cognitivas a los aportes aplicados al análisis de los procesos. En M. M., \& L. G. Meiman y M., Desarrollo Rural. Organizaciones, Instituciones y Territorio. Buenos Aires: Ciccus.

Secreto M. V. (2011). "Ese comunismo estéril en que vegetan": El individualismo agrario frente a las formas ancestrales de propiedad y los usos tradicionales de la tierra. En H. Alimomnda, La naturaleza colonizada. Ecología política y minería en America Latina. Buenos Aires: Ciccus, CLACSO.

Toledo, V. (1992). Utopía y naturaleza: El nuevo movimiento ecológico de los campesinos e indígenas de America Latina. 122, 72-85. 